\title{
Enhancement of Skin Penetration of Hydrophilic and Lipophilic Compounds by $\mathrm{pH}$-sensitive Liposomes
}

Yoshihiro Tokudome, Kaoru Nakamura, Yurina Itaya, Fumie Hashimoto

Laboratory of Dermatological Physiology, Faculty of Pharmaceutical Sciences, Josai University.

Received, February 24, 2015; Revised, June 18, 2015; Accepted, June 24, 2015; Published, July 4, 2015.

Abstract - PURPOSE: Enhance skin penetration of hydrophilic and lipophilic compounds using liposomes that are responsible to the $\mathrm{pH}$ of the skin surface. METHODS: $\mathrm{pH}$-sensitive liposomes were prepared by a thin layer and freeze-thaw method with dioleoyl phosphatidyl ethanolamine and cholesteryl hemisuccinate. Liposomal fusion with stratum corneum lipid liposomes was measured using fluorescence resonance energy transfer. Particle diameter and zeta potential of the liposomes after fusion were measured by dynamic light scattering and electrophoresis. RESULTS: Under neutral $\mathrm{pH}$ conditions, the diameter of the $\mathrm{pH}$-sensitive liposomes was $130 \mathrm{~nm}$ and their zeta potential was $-70 \mathrm{mV}$. In weakly acidic conditions, the diameter was larger than $3,000 \mathrm{~nm}$ and the zeta potential was $-50 \mathrm{mV}$. In contrast, the particle diameter and the zeta potential of the non-pH-sensitive liposomes remained constant under various $\mathrm{pH}$ conditions. A skin penetration study was performed on hairless mice skin using vertical diffusion cells, showing that the fusion ability of $\mathrm{pH}-$ sensitive liposomes was higher than that of non-pH-sensitive liposomes. In the skin penetration study was carried out using hydrophilic (calcein) and lipophilic ( $N$-(7-nitrobenz- 2-oxa-1,3-diazol-4yl)-PE) (NBD-PE) model compounds which were applied to the skin with $\mathrm{pH}$-sensitive liposomes as carrier. The fluorescent compounds contained within the $\mathrm{pH}$-sensitive liposomes permeated the skin more effectively than those within non-pH-sensitive liposomes, and this ability was further enhanced with the lipophilic compound. CONCLUSION: These studies suggest that $\mathrm{pH}$-sensitive liposomes have potential as an important tool for delivery of compounds into the skin.

This article is open to POST-PUBLICATION REVIEW. Registered readers (see "For Readers") may comment by clicking on ABSTRACT on the issue's contents page.

\section{INTRODUCTION}

The skin consists of the outer epidermis and the deeper dermis layer. From the inner layer to the outside, the epidermis consists of the stratum basale, the stratum spinosum, the stratum granulosum, and the stratum corneum (SC). The SC has an organized structure, in which keratinocytes act as "bricks" and intercellular lipids act as a "mortar-like material" (1). The stratum corneum acts as a barrier against many environmental agents, including drugs and active ingredients for cosmetics. Various studies have been conducted to find ways to improve the penetration of drugs and cosmetics (2-4). Generally, the sebum film that exists on the cutaneous surface has moisturizing and protective activities (5). Sebum and sweat coexist as a mixture and form a thin film that is weakly acidic ( $\mathrm{pH} 4.5-6.0)$ and contains lactic acid in the fatty acids and sweat $(6,7)$.

Liposomes are lipid bilayer structures formed from phospholipids when the latter, taken from a biological membrane, are added to an aqueous solution. Because hydrophobic and hydrophilic compounds can be held stably in either the lipid bilayer or an inner phase, liposomes are effective as drug carriers (8-10). Liposomes with various characteristics can be prepared by changing the type of lipids involved (11).

The use of liposomes as a transdermal drug carrier was reported by Mezei and Gulasekharam. (12). Additionally, transfersome (13), niosome (14), ethosome (15) were recently reported as carriers that improve transdermal drug delivery. These liposomes increased membrane fluidity and flexibility by modification of the lipids. We proposed that liposomes functioning through a different mechanism than high membrane fluidity and flexibility may be useful as a transdermal drug career. We therefore considered a liposomal preparation aimed at a high penetration into the stratum corneum

Corresponding Author: Yoshihiro Tokudome, Ph.D.; Faculty of Pharmaceutical Sciences, Josai University 1-1 Keyakidai, Sakado, Saitama, Japan; Email address: tokudome@josai.ac.jp 
intercellular lipids. To enhance skin penetration of the drug, we used liposomes that have a high affinity for the stratum corneum rather than ultradeformable liposomes.

$\mathrm{pH}-$ Sensitive liposomes can fuse with the cell membrane in response to $\mathrm{pH}$ changes (16-19). Liposomal membranes are stable under neutral conditions but membrane fusion increases under acidic conditions. The sebaceous gland of the skin is weakly acidic so we hypothesize that increased delivery of compounds into the skin will be obtained with these types of liposome.

This manuscript presents the first study on the preparation and use of $\mathrm{pH}$-sensitive liposomes for skin penetration. We measured the ability of $\mathrm{pH}$ sensitive liposomes to fuse with the stratum corneum in vitro by fluorescence resonance energy transfer. Additionally, we evaluated the skin penetration enhancement of calcein as a hydrophilic model compound and ( $\mathrm{N}$-(7-nitrobenz- 2-oxa-1,3-diazol4yl)-PE) (NBD-PE) as a lipophilic model compound, entrapped in $\mathrm{pH}$-sensitive liposomes that had been prepared using L- $\square$-dioleoyl phosphatidyl ethanolamine (DOPE) and cholesteryl hemisuccinate (CHEMS), respectively. The results demonstrate the usefulness of $\mathrm{pH}$-sensitive liposomes for skin penetration.
Materials and Methods

\section{Materials}

DOPE was purchased from NOF Corporation (Tokyo, Japan). CHEMS, $N$-(7-nitrobenz- 2-oxa1,3-diazol-4yl)-PE (NBD-PE) and $N$-(lissamine Rhodamine B-sulfonyl)-PE (Rh-PE) were purchased from Avanti Polar Lipid (Alabaster, AL, USA). Ceramide types II and III (CER NS and NP) were purchased from Matreya (Pleasant Gap, PA, USA). Cholesterol (CHOL), Palmitic acid (PA), and cholesterol sulfate (CS) were purchased from Sigma Aldrich (St. Louis, MO, USA). Calcein (sodium salt) and Tween-80 were purchased from TCI (Tokyo, Japan). Triton X-100 was purchased from Alfa Aesar (Ward Hill, MA, USA). Phosphate buffered saline (PBS) was purchased from Takara Bio Inc. (Otsu, Shiga, Japan). Chloroform and methanol were purchased from Wako Pure Chemical Industries (Osaka, Japan). The chemical structures of DOPE and CHEMS are shown in Figure 1.

\section{DOPE}

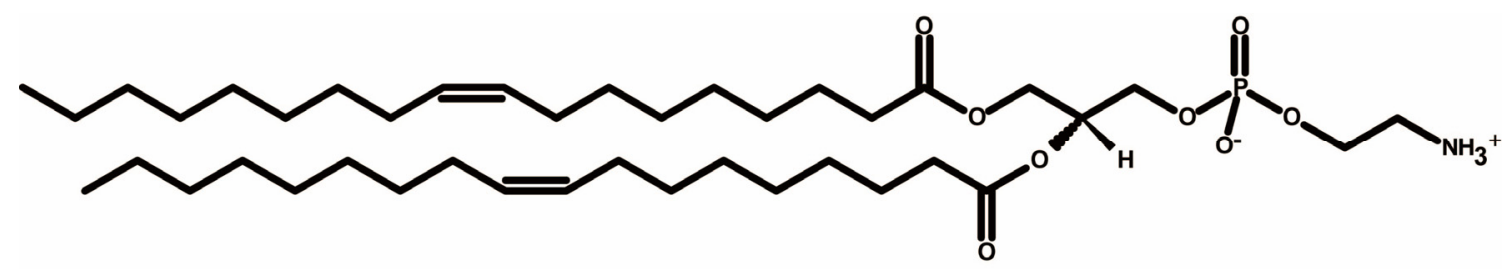

CHEMS

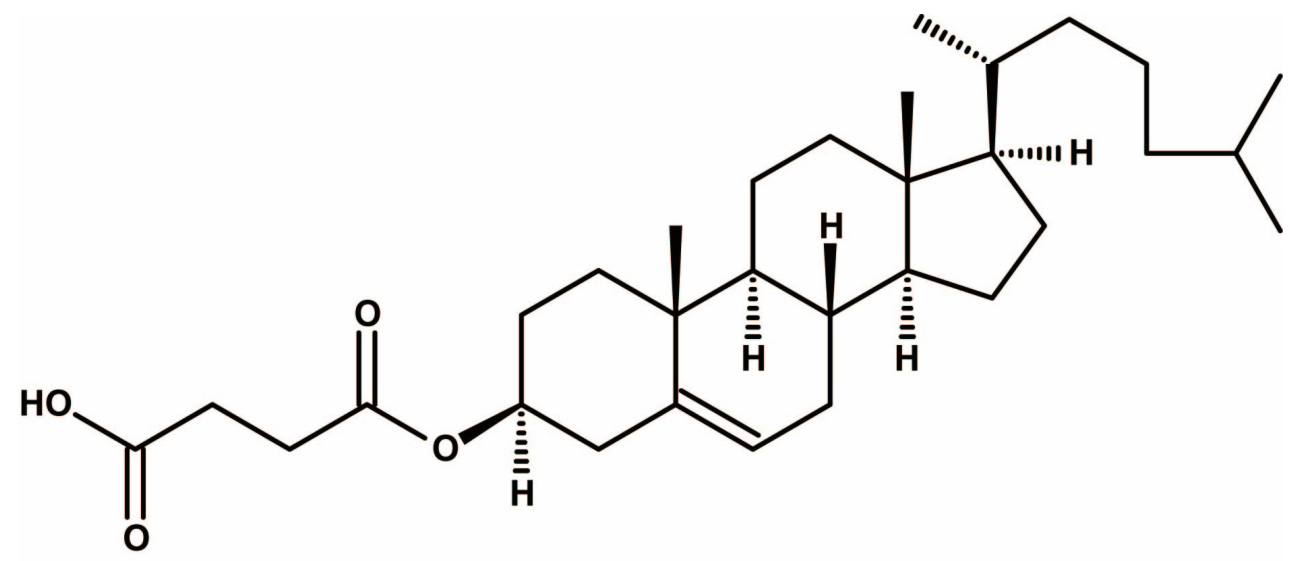

Figure 1. Dioleoyl phosphatidyl ethanolamine (DOPE) and cholesterol hemisuccinate (CHEMS). 
Preparation of $\mathrm{pH}$-sensitive and non-pH-sensitive liposomes

$\mathrm{pH}$-sensitive liposomes were composed of DOPE:CHEMS at a molar ratio of 6:4 and non-pHsensitive liposomes were composed of DOPE:CHOL at a molar ratio of 6:4. The lipids in chloroform were dried in a rotary evaporator and the lipid film was then hydrated with PBS. The liposomes were subjected to freeze-thawing for five cycles using liquid nitrogen, and extruded through a polycarbonate membrane filter with a $100-\mathrm{nm}$ pore size using an extruder (Lipex Biomembranes Inc., Vancouver, British Columbia, Canada).

Calcein entrapped liposomes were hydrated in $10 \mathrm{mM}$ calcein and the $\mathrm{pH}$ was adjusted to $\mathrm{pH} 7.4$ with sodium hydroxide. Free calcein was removed by ultracentrifugation (Himac CS 120GX II, HITACHI, Tokyo, Japan) at $113,000 \times \mathrm{g}$, at $4^{\circ} \mathrm{C}$ for 15 min. NBD-PE containing liposomes were hydrated with PBS.

\section{Preparation of SCLL (stratum corneum lipid liposomes)}

Stratum corneum lipid liposomes (SCLL) were composed of CER II:CER III:CHOL:PA:CS = 20:20:25:25:10 (w/w \%) (20). CER II, CER III, CHOL, and PA were dissolved in chloroform. CS was dissolved in chloroform:methanol (2:1). The lipids in either chloroform or chloroform:methanol $(2: 1)$ were dried in a rotary evaporator and the lipid film was then hydrated with PBS (pH 7.4). The liposomes were subjected to freeze-thawing for five cycles using liquid nitrogen and extruded through a polycarbonate membrane filter with a $100-\mathrm{nm}$ pore size using the extruder, as above.

\section{Zeta potential and particle diameter measurements}

The zeta potential and particle diameter for $\mathrm{pH}-$ sensitive liposomes, non- $\mathrm{pH}$-sensitive liposomes, and for SCLL at pH 5.0, 6.0, and 7.4, respectively, were determined using a Zetasizer Nano-ZS (ZEN3600, Malvern Instruments, Malvern, Worcestershire, UK).

Fluorescence resonance energy transfer measurements

Measurement of the ability of liposomes to fuse to SCLL was performed using fluorescence resonance energy transfer (FRET) $(20,21)$. Labeled SCLL were prepared with Rh-PE and NBD-PE at a final concentration of $1 \mathrm{~mol} \%$. Labeled SCLL $(60 \mu \mathrm{L})$ and unlabeled liposomes $(160 \mu \mathrm{L})$ were mixed and the fluorescence of the donor NBD-PE (ex: $470 \mathrm{~nm}$, em: $535 \mathrm{~nm}$ ) was followed for $15 \mathrm{~min}$ on a SpectraMax $\mathrm{M} 2^{\mathrm{e}}$ micro plate reader (Molecular Devices, Sunnyvale, CA, USA). At the end of each measurement, the vesicles were disrupted with 20 $\mu \mathrm{L}$ of $10 \%(\mathrm{w} / \mathrm{v})$ TritonX-100. Lipid mixing as a percentage of infinite probe dilution was determined using the following equation: liposome fusion ability $(\%)=\left(\mathrm{F}_{\mathrm{t}}-\mathrm{F}_{0}\right) /\left(\mathrm{F}_{\max }-\mathrm{F}_{0}\right) \times 100 \%$, where $\mathrm{F}_{\mathrm{t}}$ is the fluorescent intensity of NBD-PE during the assay, $\mathrm{F}_{0}$ is the initial value of NBD-PE fluorescence, and $F_{\max }$ is the maximum fluorescence possible, given by infinite probe dilution in the presence of Triton X100.

\section{Animals}

HR-1 male hairless mice (body weight, 16-20g) were purchased from Japan SLC Inc. (Hamamatsu, Shizuoka, Japan). They were housed in temperturecontrolled rooms $\left(25 \pm 2^{\circ} \mathrm{C}\right)$ with a 12 -h light-dark cycle (07:00-19:00 h), and they were allowed free access to food (M.F. Oriental) and tap water for 1week. All animal experiments and maintenance were performed under conditions approved by the animal research committee of Josai University.

\section{In vitro penetration study}

Hairless mice skin was excised and excess the subcutaneous fat was carefully eliminated. The excised skin was mounted on a vertical diffusion cells (effective penetration area $1.77 \mathrm{~cm}^{2}$ ). The receiver volume was $5 \mathrm{~mL}$ of phosphate buffered saline (PBS) or PBS containing 5\% Tween-80 at $\mathrm{pH}$ 7.4. at $32{ }^{\circ} \mathrm{C}$.

In vitro skin penetration studies using calceincontaining liposomes

Calcein-containing $\mathrm{pH}$-sensitive liposomes and non$\mathrm{pH}$-sensitive liposomes were used as donor solutions. The receiver solution was $5.0 \mathrm{~mL}$ of PBS. The control was PBS containing calcein. The penetration experiments were performed for $24 \mathrm{~h}$. The skins were then removed from the diffusion cells and washed briefly with purified water. Each skin sample was cut into several pieces and then homogenized in $10 \mathrm{~mL}$ methanol. The calcein content in skin was assayed at ex:495 nm, em: $515 \mathrm{~nm}$ by a Shimadzu RF-5300 spectrofluorophotometer (Nakagyo-ku, Kyoto, Japan).

In vitro penetration studies using NBD-PEcontaining liposomes

NBD-PE contained in either $\mathrm{pH}$-sensitive-liposomes 
or non-pH-sensitive liposomes were used as donor solutions. The receiver solution was $5.0 \mathrm{~mL}$ of PBS containing 5\% Tween-80. Because NBD-PE did not dissolve in water, the control NBD-PE solution was not examined. The penetration experiments were performed for $24 \mathrm{~h}$, after which the skins were removed from the diffusion cells and washed briefly with purified water. Each skin sample was cut into several pieces and then homogenized in $10 \mathrm{~mL}$ methanol. The NBD-PE content in the skin was assayed at ex: $470 \mathrm{~nm}$, em: $535 \mathrm{~nm}$ by spectrofluorophotometer.

\section{RESULTS}

Changes in particle diameter and zeta potential of $\mathbf{p H}$-sensitive and non-pH-sensitive liposomes Immediately after sizing, the particle diameters of the $\mathrm{pH}$-sensitive and non-pH-sensitive liposomes at pH 7.4 were 130.0 and $134.3 \mathrm{~nm}$, respectively and their zeta potentials were -70.5 and $-68.6 \mathrm{mV}$, respectively. The particle diameters and zeta potentials of the liposomes did not change over the 24 hours (Fig. 2A and B).

(A)

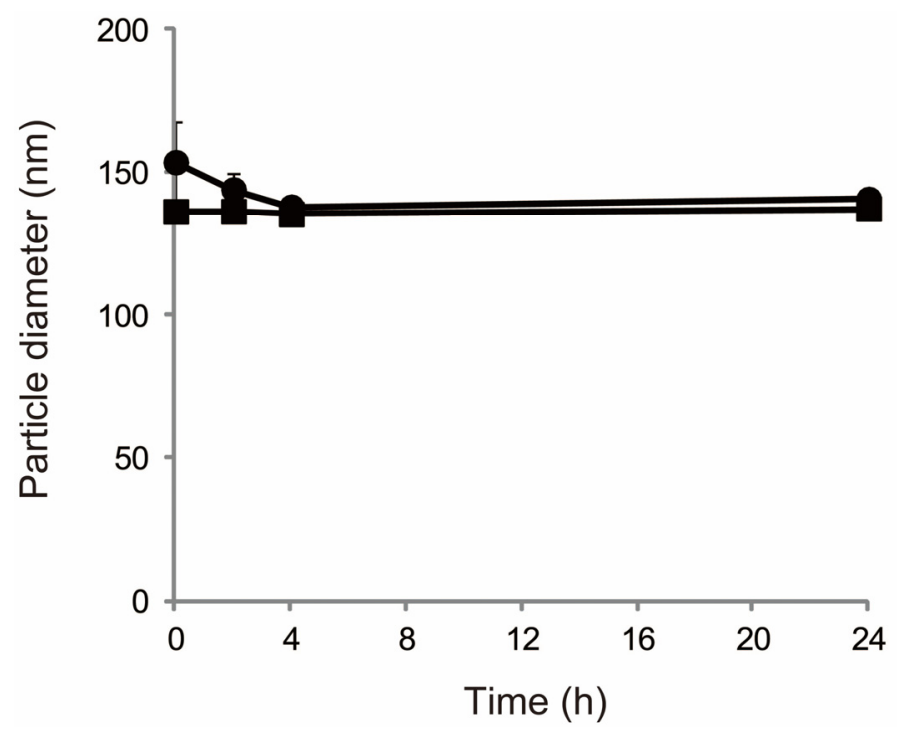

(B)

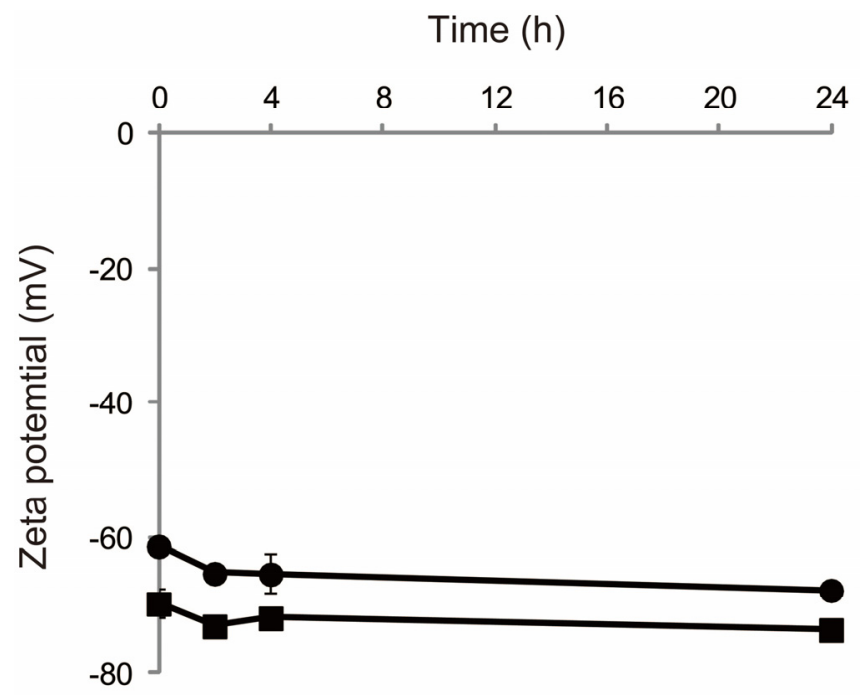

Figure 2. Changes in particle diameter (A) and zeta potential (B) of pH-sensitive (squares) and non-pH-sensitive (circles) liposome at $32^{\circ} \mathrm{C}$; mean $\pm \mathrm{SD}, \mathrm{n}=3$. 
Changes in the particle diameter and zeta potential of the $\mathrm{pH}$-sensitive and non-pH-sensitive liposomes under various $\mathrm{pH}$ conditions.

The particle diameters of SCLL and non-pHsensitive liposomes (DOPE/CHOL) were unchanged in phosphate buffer at $\mathrm{pH} 5.0,6.0$, and 7.4 (Fig. 3A). Moreover, the diameters of the $\mathrm{pH}-$ sensitive liposomes (DOPE/CHEMS) were unchanged at $\mathrm{pH} 6.0$ and 7.4. However, the particle diameter of $\mathrm{pH}$-sensitive liposomes increased only at $\mathrm{pH}$ 5.0. The zeta potentials of the $\mathrm{pH}$-sensitive and non-pH-sensitive liposomes were unchanged at $\mathrm{pH}$ 6.0 and 7.4. In contrast, the zeta potential approached neutrality only for the $\mathrm{pH}$-sensitive liposomes at $\mathrm{pH} 5.0$ (Fig. 3B).

(A)

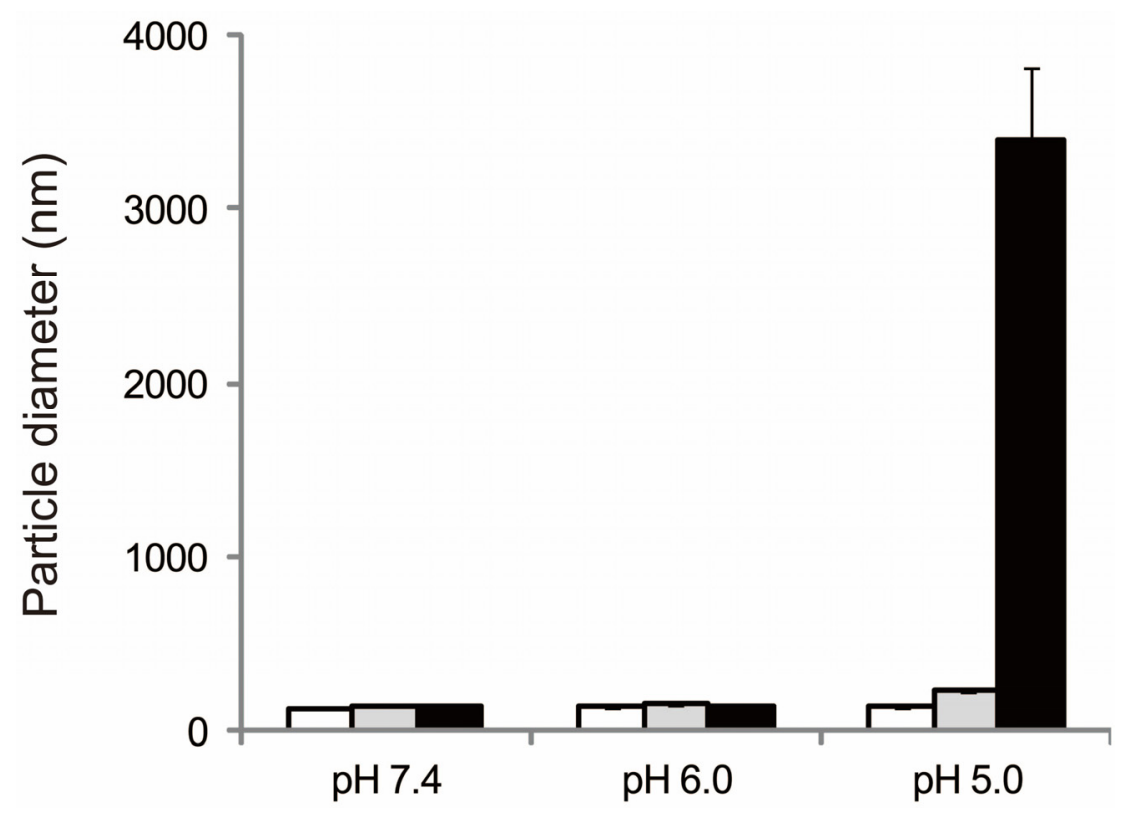

(B)

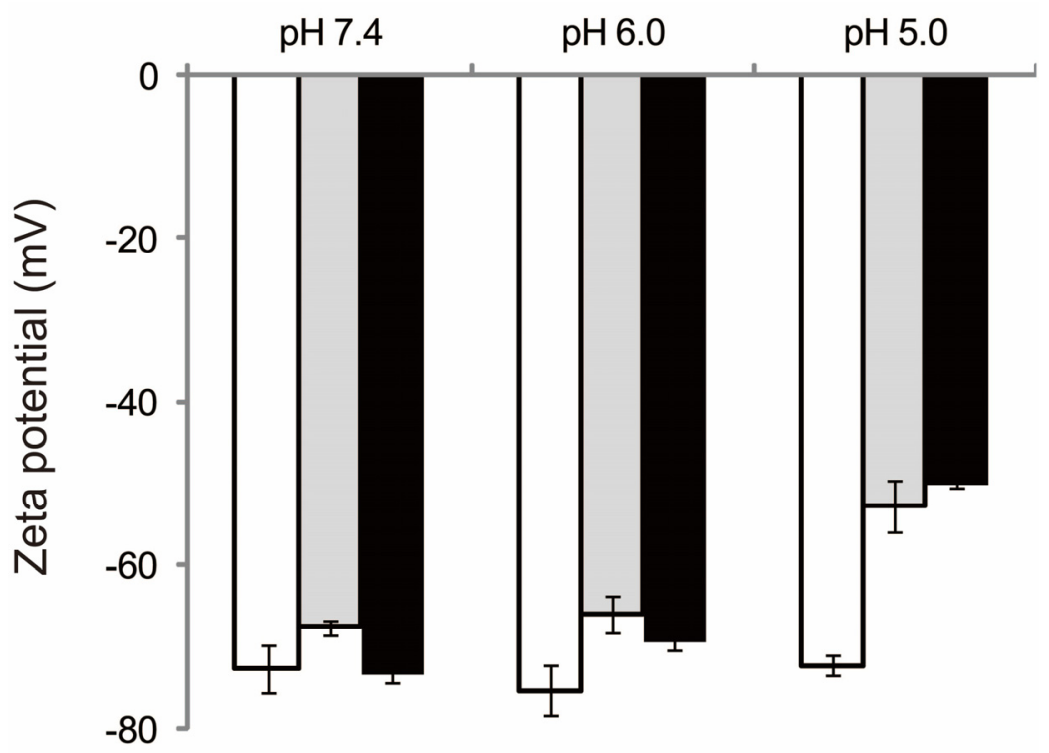

Figure 3. Particle diameter (A) and zeta potential (B) of SCLL (open bars) . non-pH-sensitive liposome (DOPE/CHOL, gray bars) and $\mathrm{pH}$-sensitive liposome (DOPE/CHEMs, filld bars); mean $\pm \mathrm{SD}, \mathrm{n}=3$. 
Evaluation of in vitro fusion ability of $\mathrm{pH}-$ sensitive and non-pH-sensitive liposomes with SCLL at pH 5.0

The abilities of $\mathrm{pH}$-sensitive liposomes and non- $\mathrm{pH}-$ sensitive liposomes to fuse with SCLL are shown in Figure 4 as $48.4 \%$ and $8.7 \%$ at 15 min, respectively. These differences were significant $(\mathrm{p}<0.001)$ and demonstrate the potentially important activity of $\mathrm{pH}-$ sensitive liposomes on drug delivery into the skin.

\section{In vitro skin penetration}

Hydrophilic compound delivery to the skin by pH-sensitive and $\mathrm{pH}$ non-pH-sensitive liposomes

The calcein concentration delivered to the skin by calcein encapsulated in $\mathrm{pH}$-sensitive and non-pHsensitive liposomes did not differ from the control but that delivered by $\mathrm{pH}$-sensitive liposomes was significantly higher ( $<<0.05$, Fig. 5), again showing the potential of the $\mathrm{pH}$-sensitive liposomes for drug delivery into the skin.

Lipophilic compound concentration delivery to the skin by $\mathrm{pH}$-sensitive and non-pH-sensitive liposomes.
The NBD-PE concentration delivered to the skin by the $\mathrm{pH}$-sensitive liposomes was significantly higher than that delivered by non-pH-sensitive liposomes $(p<0.01$, Fig. 6$)$. The NBD-PE concentrations delivered to the skin by the $\mathrm{pH}$ sensitive and the non-pH-sensitive liposomes were 35.81 and $1.79 \mathrm{pmol} / \mathrm{cm}^{2}$, respectively.

\section{DISCUSSION}

It was reported recently that ultra-deformable liposomes enhance skin penetration of drugs. Generally, flexible liposomes incorporate surfactants within their membranes and this is a general method for imparting fluidity to liposomal membranes. Instead of using elastic liposomes, we attempted to enhance skin penetration using liposomes with a high affinity for the stratum corneum. This study examined the physical properties and skin penetration activities of hydrophilic and lipophilic drugs entrapped in CHEMS- and DOPE-based $\mathrm{pH}$-sensitive liposomes.

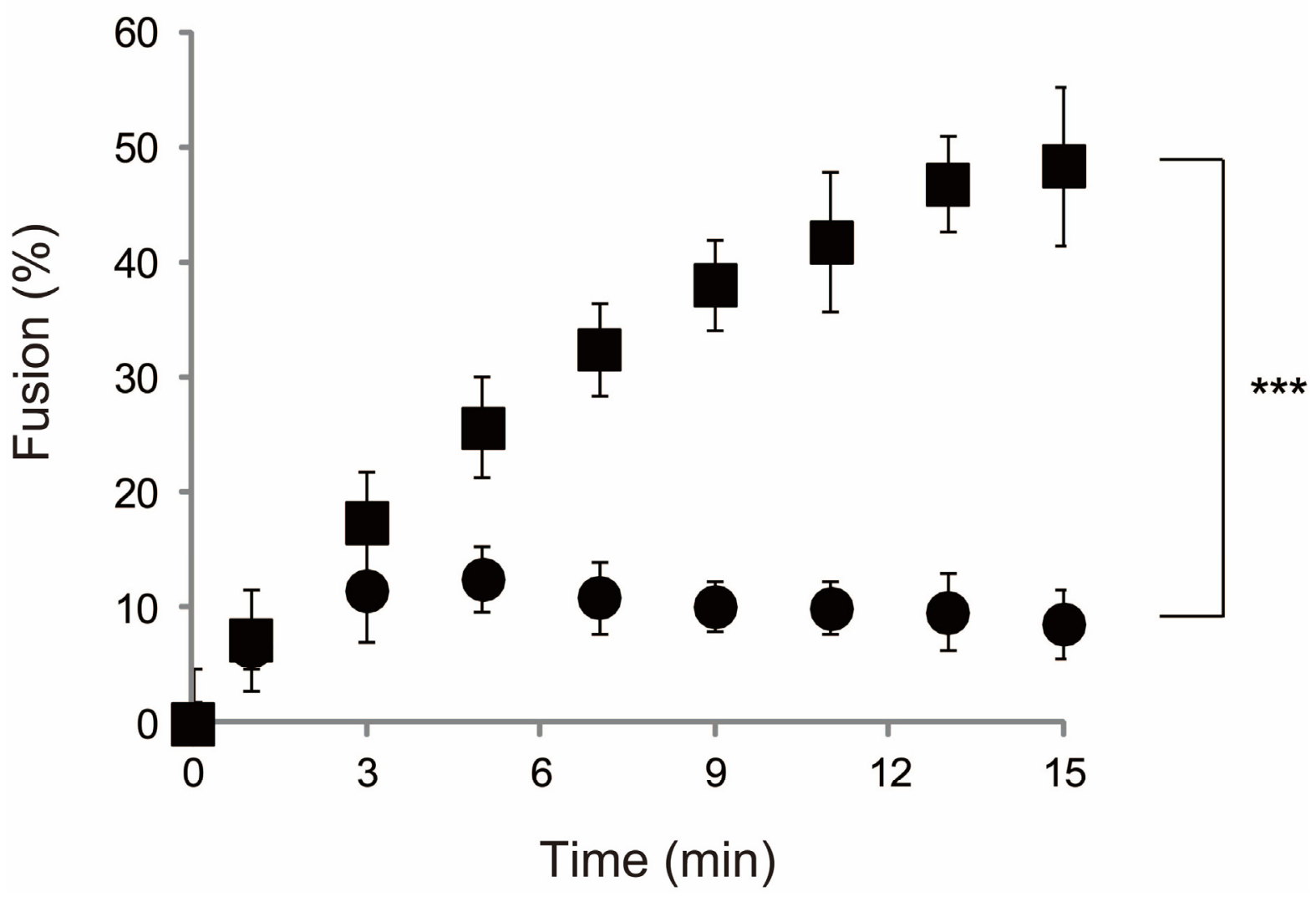

Figure 4. Ability of $\mathrm{pH}$-sensitive (filled square) and non-pH-sensitive liposomes (filled circle) to fuse with stratum corneum liposomes at $\mathrm{pH}$ 5.0. 


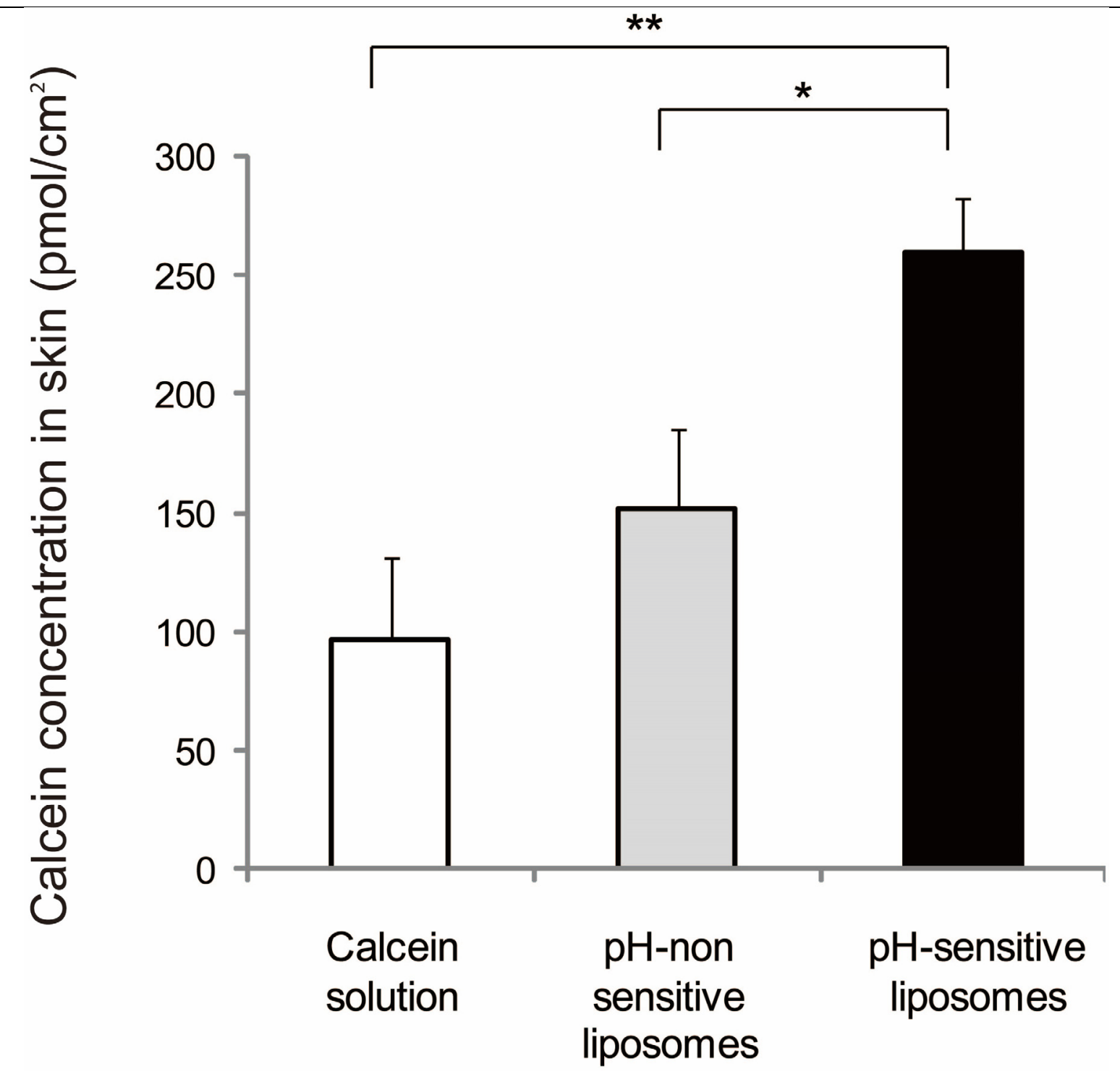

Figure 5. Skin permeation of calcein encapsulated in $\mathrm{pH}$-sensitive (filled bar) and non-pH-sensitive liposomes (gray bar), and by calcein solution alone (open bar). The data represent the average and standard deviation $(n=4)$, respectively. ${ }^{*}, \mathrm{p}<0.05, * *, \mathrm{p}<0.01$, Tukey's multiple comparison test.

In terms of physical properties, the particle diameters and zeta-potentials of $\mathrm{pH}$-sensitive (DOPE/CHEMS) liposomes and non-pH-sensitive liposomes were constant at $\mathrm{pH} 6.0$ and 7.4. In contrast, the particle diameters changed significantly at $\mathrm{pH}$ 5.0, and the zeta potentials were lower for both types of liposomes. The increase in liposome particle diameters is thought to be caused either by fusion of liposomes to one another or by the instability of the liposomes themselves. CHEMS becomes partially protonated under acidic conditions. Therefore, we suggest that the negative charge is lost under acidic conditions and DOPE becomes a reversed micelle structure because of the loss of repulsion between the polar groups. Therefore, the electric charge reverses, and it is likely that the liposomal zeta potential was inclined towards neutral.

In our study, FRET was used to measure the fusion ability $(20,21)$. FRET involves excitation energy being transferred directly by electronic resonance between two adjoining fluorescent molecules. When NBD-PE (excitation: $470 \mathrm{~nm}$, emission: $535 \mathrm{~nm}$ ) and Rh-PE (excitation: $575 \mathrm{~nm}$, emission: $589 \mathrm{~nm}$ ) coexist in liposomes, this allows most of the fluorescence of NBD-PE to be quenched by the excitation energy transfer. 


\section{$* *$}

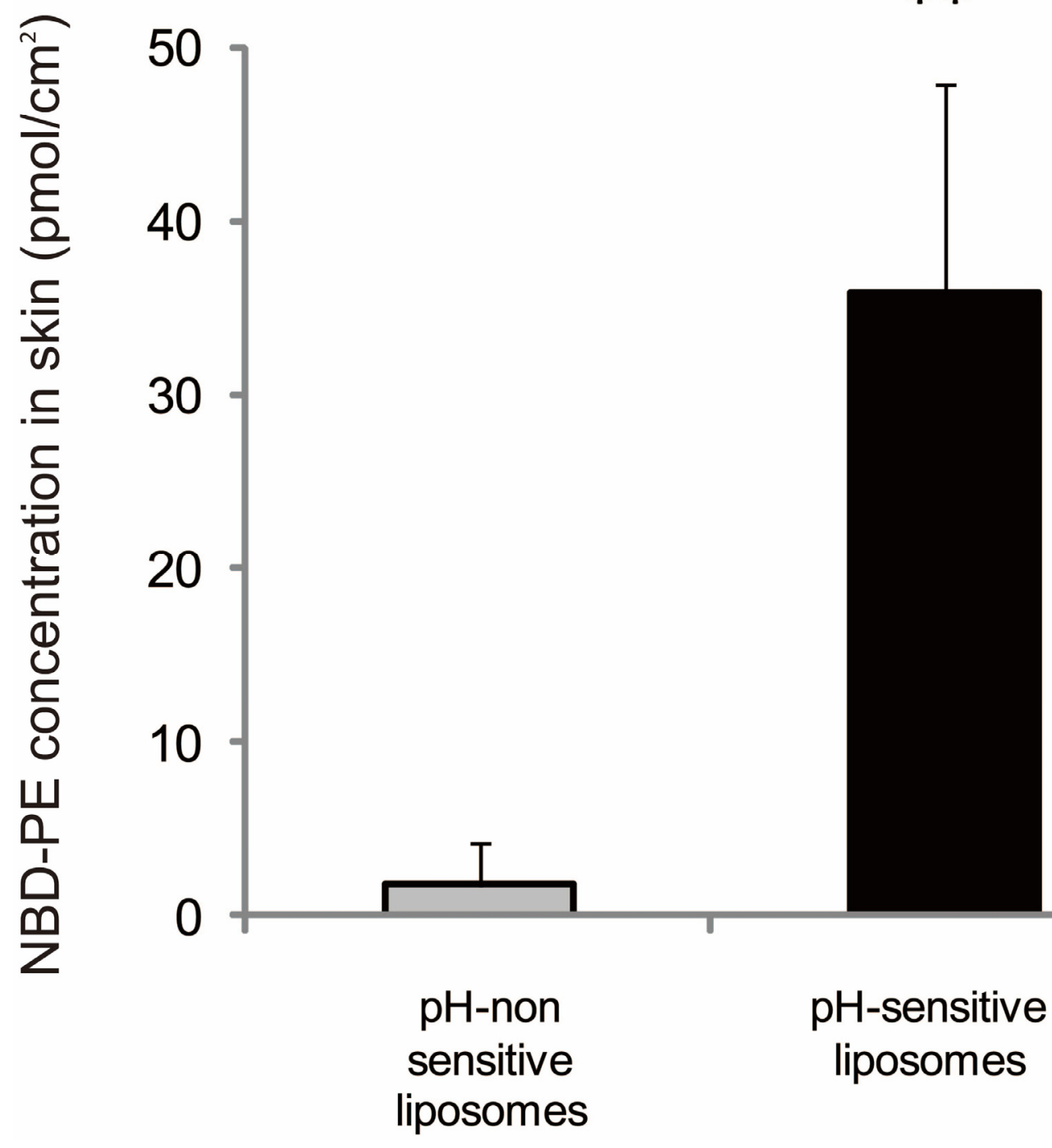

Figure 6. Skin permeation of NBD-PE encapsulated in $\mathrm{pH}$-sensitive (filled bar) and non-pH-sensitive liposomes (gray bar). The data represent the average and standard deviation $(n=4)$, respectively. ${ }^{* * *}, \mathrm{p}<0.001$ compared with the nonpH-sensitive liposomes (Student's statistical test).

When two fluorescence compounds are contained in liposomes that fuse with non-labeled liposomes, the concentration of fluorescent probes decreases. Therefore, the fluorescence quenching of NBD-PE can be recovered and the membrane fusion ability can be evaluated by measuring this. $\mathrm{pH}$-sensitive liposomes showed a high fusion activity for SCLL compared with non-pH-sensitive liposomes at $\mathrm{pH}$ 5.0. Because $\mathrm{pH}$-sensitive liposomes fuse easily with SCLL under acidic conditions, it was assumed that the skin will also show a high fusion ability. Therefore, an in vitro skin penetration study was performed.
Calcein was entrapped as a hydrophilic model drug in $\mathrm{pH}$-sensitive and non-pH-sensitive liposomes. The in vitro skin concentration resulting from calcein entrapped in $\mathrm{pH}$-sensitive liposomes was significantly higher than that from non-pHsensitive liposomes. The in vitro skin concentration of NBD-PE as a hydrophilic model compound entrapped in pH-sensitive liposomes was significantly higher than that of non-pH-sensitive liposomes. Under acidic conditions, DOPE is thought to have a reversed micelle structure $(22,23)$ and to be able to fuse with the membrane. Subsequently, release of the entrapped compound can occur, resulting in enhanced skin penetration. 
In conclusion, $\mathrm{pH}$-sensitive liposomes are a unique and important approach to increasing skin penetration and delivery of hydrophilic and lipophilic compounds or drugs.

Declaration of interest: The authors report no financial conflicts of interest. The authors alone were responsible for the content and writing of this paper.

\section{REFERENCES}

1. Elias PM, Epidermal lipids, barrier function, and desquamation. J Invest Dermatol, 1983; 80: (suppl) 44-49.

2. Otto A, Wiechers JW, Kelly CL, Hadgraft J, du Plessis J. Effect of penetration modifiers on the dermal and transdermal delivery of drugs and cosmetic active ingredients. Skin Pharmacol Physiol, 2008; 21: 326-334.

3. Marianecci C, Rinaldi F, Esposito S, Di Marzio L, Carafa M. Niosomes encapsulating Ibuprofencycrodextrin complex: preparation and characterization. Curr Drug Targets, 2013; 14: 10701078.

4. Zillich OV, Schweiggert-Weisz U, Hasenkopf $\mathrm{K}$, Eisner P, Kerscher M. Release and in vitro skin permeation of polyphenols from cosmetic emulsions. Int J Cosmet Sci, 2013; 35: 491-501.

5. Schneider MR, Paus R. Sebocytes, multifaceted epithelial cells: lipid production and holocrine secretion. Int J Biochem Cell Biol, 2010; 42: 181-185.

6. Chan A, Mauro T. Acidification in the epidermis and the role of secretory phospholipases. Dermatoendocrinol, 2011; 3: 84-90.

7. Elias PM. Stratum corneum defensive functions: an integrated view. J Invest Dermatol, 2005; 125: 183200.

8. Shmeeda H, Amitay Y, Tzemach D, Gorin J, Gabizon A. Liposome encapsulation of zoledronic acid results in major changes in tissue distribution and increase in toxicity. J Control Release, 2013; 167: 265-275.

9. Nie Y, Ji L, Ding H, Xie L, Li L, He B, Wu Y, Gu Z. Cholesterol derivatives based charged liposomes for doxorubicin delivery: preparation, in vitro and in vivo characterization. Theranostics, 2012; 2: 10921103.

10. Odeh F, Ismail SI, Abu-Dahab R, Mahmoud IS, Al Bawab A. Thymoquinone in liposomes: a study of loading efficiency and biological activity towards breast cancer. Drug Deliv, 2012; 8: 371-377.

11. Villasmil-Sánchez S, Drhimeur W, Ospino SC, Rabasco Alvarez AM, González-Rodríguez ML. Positively and negatively charged liposomes as carriers for transdermal delivery of sumatriptan: in vitro characterization. Drug Dev Ind Pharm, 2010;
36: 666-675.

12. Mezei M, Gulasekharam V. Liposomes- a selective drug delivery system for the topical route of administration. Lotion dosage form. Life Sci, 1980; 26: 1473-1477.

13. Cevc G, Blume G. Lipid vesicles penetrate into intact skin owing to the transdermal osmotic gradients and hydration force. Biochim Biophys Acta, 1992; 1104: 226-232.

14. Schreier H, Bouwstra J. Liposomes and niosomes as topical drug carriers: dermal and transdermal drug delivery. J Control Release, 1994; 30: 1-15.

15. Touitou E, Dayan N, Bergelson L, Godin B, Eliaz M. Ethosomes - novel vesicular carriers for enhanced delivery: characterization and skin penetration properties. J Control Release, 2000; 65: 403-418.

16. Connor J, Yatvin MB, Huang L. pH-sensitive liposomes: acid-induced liposome fusion. Proc Natl Acad Sci USA, 1984; 81: 1715-1718.

17. Tachibana R, Harashima H, Shono M, Azumano M, Niwa M, Futaki S, Kiwada H. Intracellular regulation of macromolecules using $\mathrm{pH}$-sensitive liposomes and nuclear localization signal: qualitative and quantitative evaluation of intracellular trafficking. Biochem Biophys Res Commun, 1998; 251: 538-544.

18. Hafez IM, Cullis PR. Cholesteryl hemisuccinate exhibits $\mathrm{pH}$ sensitive polymorphic phase behavior. Biochim Biophys Acta, 2000; 1463: 107-114.

19. Vanić Z, Barnert S, Süss R, Schubert R, Fusogenic activity of PEGylated pH-sensitive liposomes. J Liposome Res, 2012; 22: 148-157.

20. Tokudome Y, Saito Y, Sato F, Kikuchi M, Hinokitani $\mathrm{T}$, Goto K, Preparation and characterization of ceramide-based liposomes with high fusion activity and high membrane fluidity. Colloids and Surfaces B : Biointerfaces, 2009; 73: 92-96.

21. Hayashi K, Tatsui T, Shimanouchi T, Umakoshi H. Membrane interaction between Span 80 vesicle and phospholipid vesicle (liposome): Span 80 vesicle can perturb and hemifuse with liposomal membrane. Colloids Surf. B Biointerfaces, 2013; 106: 258-264.

22. Sánchez M, Aranda FJ, Teruel JA, Ortiz A. New pHsensitive liposomes containing phosphatidylethanolamine and a bacterial dirhamnolipid. Chem Phys Lipids, 2011; 164: 16-23.

23. Sudimack JJ, Guo W, Tjarks W, Lee RJ, A novel pHsensitive liposome formulation containing oleyl alcohol. Biochim. Biophys. Acta, 2002; 1564: 31-37. 